\title{
Influence of the blood exposure time in dynamic hemocompatibility testing on coagulation and C5a activation
}

\author{
Bernhard Hiebl $^{\mathrm{a}, *}$, Nico Scharnagl ${ }^{\mathrm{b}}$, Sabine Kaessmeyer ${ }^{\mathrm{c}}$, Ole Gemeinhardt ${ }^{\mathrm{d}}$, \\ Ines Gemeinhardt ${ }^{\mathrm{e}}$, Stefan M. Niehues ${ }^{\mathrm{e}}$, Sinem Peters ${ }^{\mathrm{f}}$ and Friedrich Jung ${ }^{\mathrm{g}}$ \\ ${ }^{a}$ Center for Medical Basic Research, Martin-Luther-University Halle-Wittenberg, Halle (Saale), \\ Germany \\ ${ }^{\mathrm{b}}$ Institute of Materials Research, Magnesium Innovations Center, Helmholtz-Zentrum Geesthacht, \\ Zentrum für Material- und Küstenforschung GmbH, Geesthacht, Germany \\ ${ }^{\mathrm{c}}$ Freie Universität Berlin, Department of Veterinary Medicine, Institute of Veterinary Anatomy, Berlin, \\ Germany \\ ${ }^{\mathrm{d}}$ Charité-University Medicine Berlin, Department of General, Visceral and Vascular Surgery, \\ Berlin, Germany \\ ${ }^{\mathrm{e}}$ Charité-University Medicine Berlin, Department of Radiology, Berlin, Germany \\ ${ }^{\mathrm{f}} \mathrm{CREAMEDIX} \mathrm{GmbH}$, Weingarten, Germany \\ ${ }^{\mathrm{g}}$ Institute of Biomaterial Science and Berlin-Brandenburg Center for Regenerative Therapies, \\ Helmholtz-Zentrum Geesthacht, Teltow, Germany
}

\begin{abstract}
Within the hemocompatibility testing portfolio of medical devices a range of dynamic models were established in recent years. In contrast to the static hemocompatibility testing method the dynamic models allow considering the impact of hemorheological and hemodynamic blood characteristics on the hemocompatibility of medical devices. Unfortunately the EN DIN ISO 10993-4 for the biological evaluation of medical devices for interaction with blood gives no hints towards the period of time during which the medical devices should be exposed to the blood in these tests. To examine whether different exposure times impact the comparability of hemocompatibility test results low density polyethylene (LD-PE) tubes and nitinol stents were tested exemplarily in a closed loop model for changes of the fibrinogen content, the prothrombin time, the thrombin time, and the C5a activity after 30 and $90 \mathrm{~min}$ exposure to the blood. Low density polyethylene was used as negative control because it is one of the European reference materials for hemocompatibility testing. After 90 min blood exposure to the LD-PE tubing and the nitinol stents the prothrombin time was significantly longer and the fibrinogen content significantly lower $(p<0.05)$ than after $30 \mathrm{~min}$. In contrast the thrombin time and the C5a were comparable after 30 and $90 \mathrm{~min}$ blood exposure time. These results might recommend to an initial $30 \mathrm{~min}$ exposure time, which is followed by a 90 min exposure time in cases of unclear results.
\end{abstract}

Keywords: Hemocompatibility, biocompatibility, complement, C5a, coagulation, biomaterials

\section{Introduction}

Medical devices where biomaterials contact the blood of patients (e.g. catheters, blood vessel grafts, vascular stents, artificial heart valves, circulatory support devices, various extracorporeal tubing, hemodialysis, hemapheresis, and oxygenator membranes) are applied in enormous quantities. A safe and sufficient application of these medical devices and the respective biomaterials being in contact

\footnotetext{
*Corresponding author: Bernhard Hiebl, Center for Medical Basic Research, Martin-Luther-University Halle-Wittenberg, Halle (Saale), Germany. E-mail: bernhard.hiebl@medizin.uni-halle.de.
} 
with blood either temporarily or permanently has to provide hemocompatibility. Hemocompatibility is defined as a lack of significant adverse interactions of a substrate with the found elements of the blood reactions [1]. The blood reactions are initiated by physical and chemical characteristics of the foreign surface. In vivo hemocompatibility is mediated by the glycocalix of endothelial cells contiguously lining the luminal surface of blood vessels. When biomaterials come in contact with blood the interaction between both can range from minimal protein adsorption to activation of coagulation, complement and destruction of cells. From the ISO standards perspective, five test categories are indicated for hemocompatibility evaluation: thrombosis, coagulation, platelets, haematology, and immunology (complement and leukocytes) (EN DIN ISO 10993-4). Unfortunately there is still a lack in standardized and generally accepted test procedures in order to quantify and validate precisely the events on the blood-biomaterial interface [2-4]. For medical devices and biomaterials which are developed for usage under flow conditions dynamic hemocompatibility test settings are established [5]. However, up to now there is no recommendation for the time period in which medical devices or the respective biomaterials have to be exposed to blood or blood components in these dynamic test settings. Under physiologic conditions the clotting process on biomaterial interfaces takes up to 48 min until fibrinolysis starts [6] and there are different studies recommending a maximal exposure time of the blood with the test samples of $4 \mathrm{hrs}$. Otherwise the functional integrity of the blood cells and proteins might be affected [7-11]. The study therefore aimed to investigate the influence of two time periods of blood exposure to medical devices (30 min and $90 \mathrm{~min}$ ) on coagulation and complement activation to support standardisation of dynamic hemocompatibility test setups.

\section{Materials and methods}

\subsection{Blood sampling}

The blood donors $(n=4)$ were thoroughly informed about the aims and risks of the examination and they gave their written consent [12].

$50 \mathrm{ml}$ of venous blood were drawn in a standardized manner - stratified according to the Nordkem workshop criteria - from apparently healthy subjects $[13,14]$ and anticoagulated with Na-Citrate. Single use syringes and a butterfly system with a cannula size of $21 \mathrm{G}$ were applied to draw the blood from the cubital veins [15]. The butterfly system was flushed with the anticoagulant before usage, and the syringe was prefilled with the anticoagulant ( $1 \mathrm{ml} \mathrm{Na}$-citrate $/ 9 \mathrm{ml}$ blood). During blood sampling it was taken care to use only gently aspiration and to mix the blood with the anticoagulant already during the aspiration phase by turning the syringe.

\subsection{Dynamic hemocompatibility test system}

Tests were performed using an in vitro closed loop model. The system consists of a roller pump (Watson-Marlow 101F/R, UK), in which a medical grade silicone tube (Versilic ${ }^{\mathrm{TM}}$, length $24.0 \mathrm{~cm}$, ID $4.0 \mathrm{~mm}$, OD $6.0 \mathrm{~mm}$ ) was placed. The ends of this tube were connected to separate polyvinylidenfluorid based three-way valves. Later were connected together by a further silicone tube (Versilic ${ }^{\mathrm{TM}}$, length $6.5 \mathrm{~cm}$, ID $5.0 \mathrm{~mm}$, OD $8.0 \mathrm{~mm}$ ) into which the low density polyethylene tube (LD-PE, ID $3 \mathrm{~mm}$, OD $5 \mathrm{~mm}$, RCT Reichelt Chemietechnik, Germany) or the stent were inserted. LD PE is recommended as negative control material for hemocompatibility testings in the EN DIN ISO 10993-12 and the European Concerted Action "EUROBIOMAT - Hemocompatibility" [16].

All blood connecting parts of the closed loop system were only used after sterilization by autoclaving $\left(121^{\circ} \mathrm{C}, 20 \mathrm{~min}\right)$. The tests were performed at body temperature $\left(37^{\circ} \mathrm{C}\right)$. To 


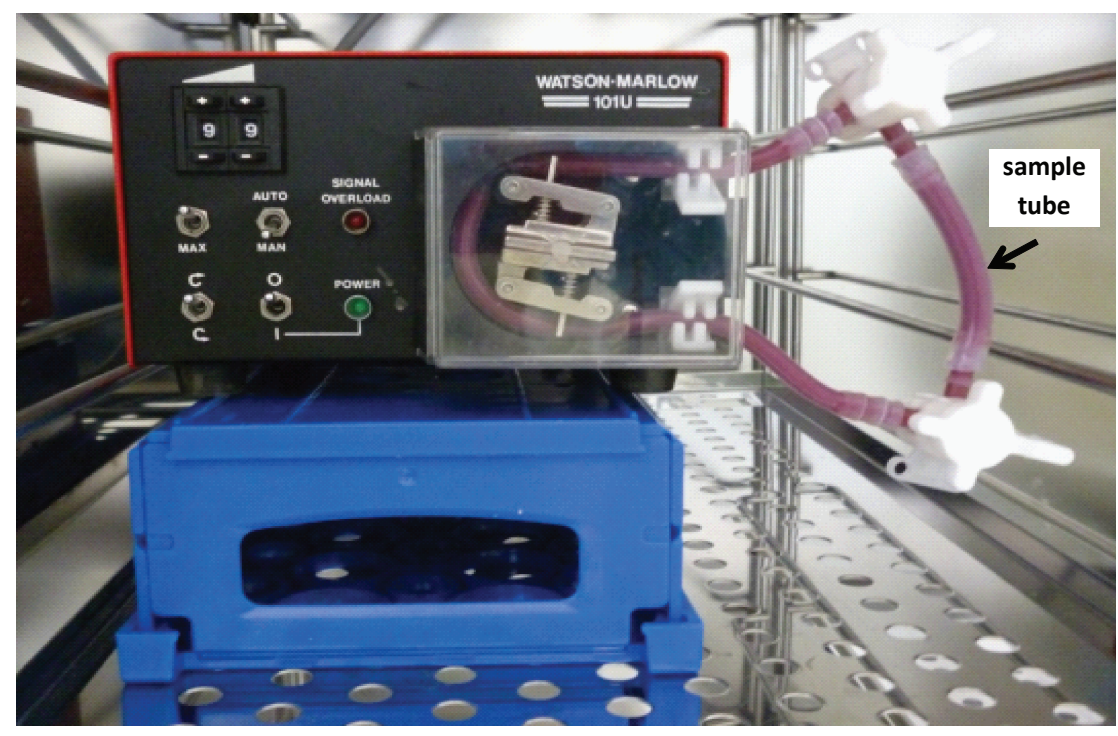

Fig. 1. Heating cabinet with a closed loop system inside, consisting of a silicon tube based circuit which is placed in a roller pump. The sample is inserted into the sample tube.

guarantee constant temperature conditions the closed loop system was placed in a heating cabinet (Fig. 1).

After filling the system via the three-way valves with anticoagulated blood the running roller pump maintained a blood flow of $40 \mathrm{ml} / \mathrm{min}$ within the circuit. After $30 \mathrm{~min}$ and $90 \mathrm{~min}$ the blood flow was stopped.

The blood was thereafter transferred into medical grade and sterile polypropylene based centrifuge tubes and centrifuged at $2500 \mathrm{~g}$ at room temperature for $15 \mathrm{~min}$ for plasma separation. The plasma was processed by a second centrifugation step ( $2500 \mathrm{~g}, 15 \mathrm{~min}$ ) before usage.

\subsection{Complement activity}

The influence of the time period of testing on the complement system was analyzed by measuring plasma C5a which is a key element within the complement cascade (C5a MicroVue ${ }^{\mathrm{TM}}$ Quidel ELISA-Kit, TecoMedical). The test was performed in strong accordance to the manufacturer's instructions.

\subsection{Coagulation}

The influence of different testing periods on the coagulation system was assessed by measuring the fibrinogen content of the plasma, the prothrombin time (PT) and the thrombin time (TT) with a coagulation analyzer (Sysmex CA-540, Siemens Healthcare Diagnostics).

\subsection{Statistics}

Data were reported as mean value \pm standard deviation for continuous variables, and were analyzed by Student's $t$-test (paired). A $p$ value of less than 0.05 was considered significant. 


\section{Results}

The time period of testing influenced significantly the coagulation parameters prothrombin time and fibrinogen content. After 90 min the prothrombin time was significantly higher $(p<0.05)$ than after 30 min (Fig. 2).

The prolonged prothrombin time after the 90 min lasting test cycle was accompanied by a lower fibrinogen concentration in comparison to the test period of $30 \mathrm{~min}(p<0.05$, Fig. 3$)$.

In contrast to the prothrombin time and the fibrinogen concentration the thrombin time showed no differences after the different time periods of testing (30 min and $90 \mathrm{~min}$, Fig. 4).

The cleavage of the complement factor C5 by the C5 convertase into C5a is a key step within the complement cascade. After exposure of the LD-PE in the closed loop system the 90 min lasting time period of testing resulted in a comparable C5a content as after $30 \mathrm{~min}$ (Fig. 5).

\section{Discussion}

The study was aimed to standardize dynamic hemocompatibility testing models by assessing the impact of two different time periods of testing on the parameters of coagulation and on the complement

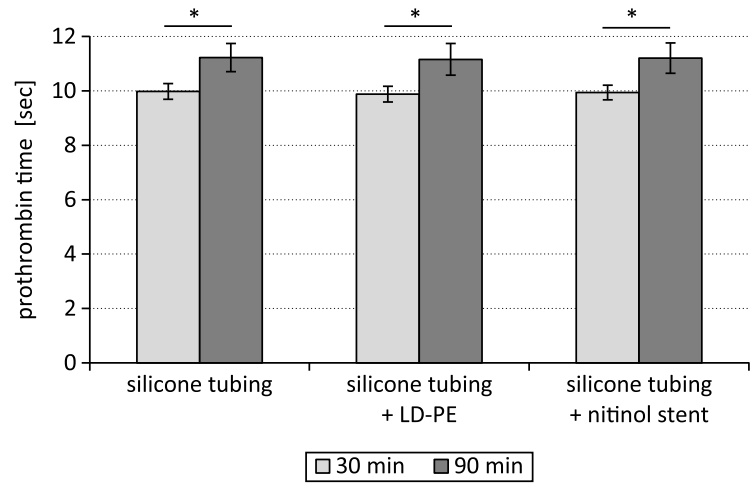

Fig. 2. Prothrombin time after testing a silicone tubing with integrated samples (LD-PE: low density polyethylene tube, nitinol stent) in a closed loop model; $* p<0.05, n=6$.

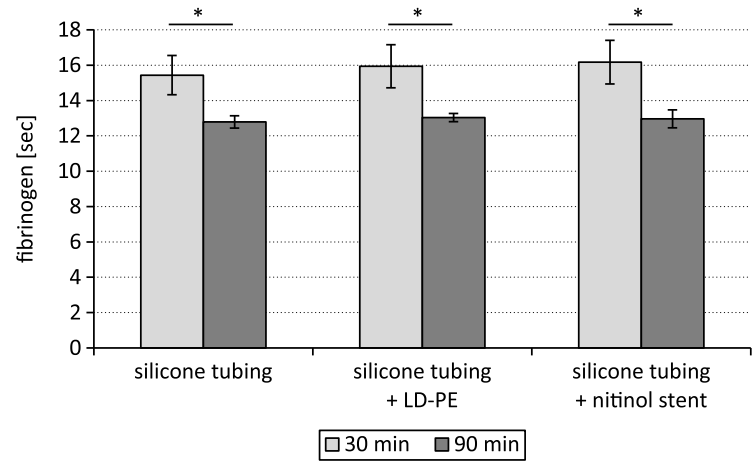

Fig. 3. Fibrinogen content after testing a silicone tubing with integrated samples (LD-PE: low density polyethylene tube, nitinol stent) in a closed loop model; $* p<0.05, n=6$. 


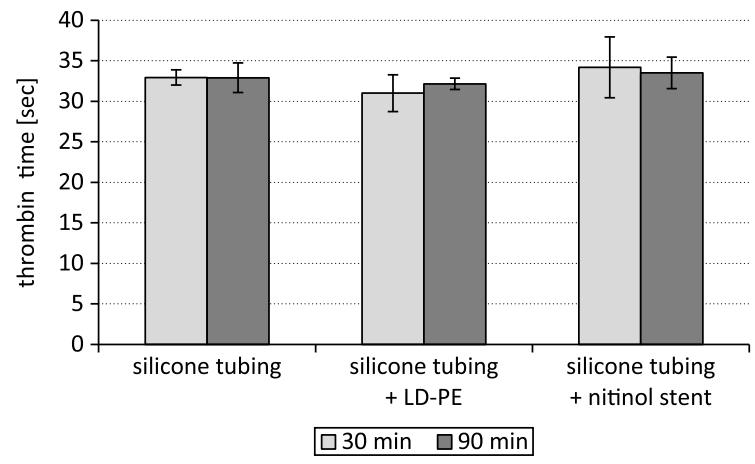

Fig. 4. Thrombin time after testing a silicone tubing with integrated samples (LD-PE: low density polyethylene tube, nitinol stent) in a closed loop model; $n=6$.

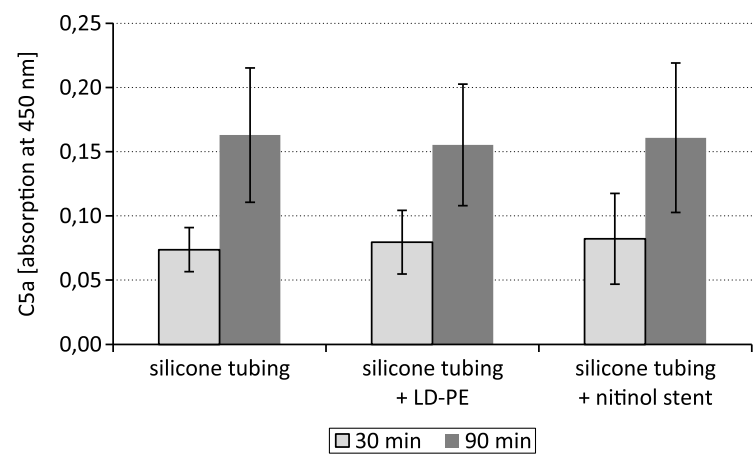

Fig. 5. C5a content after testing a silicone tubing with integrated samples (LD-PE: low density polyethylene tube, nitinol stent) in a closed loop model; $n=6$.

system. The results showed that the concentration of the complement system activation marker C5a was not different between the $30 \mathrm{~min}$ and $90 \mathrm{~min}$ time period of testing.

In the course of complement activation either via the classical or the alternative pathway the anaphylatoxic peptide $\mathrm{C} 5 \mathrm{a}$ is generated by the proteolytic cleavage of the respective parent molecule $\mathrm{C} 5$. There are four different C5 convertases which are known to cause proteolytic cleavage of the C5 glycoprotein into C5a and C5b fragments. All these enzymes are unstable and undergo decay dissociation in biological fluids. The cell surface associated complement enzymes $\mathrm{C} 4 \mathrm{~b} 2 \mathrm{a} 3 \mathrm{~b}$ and $\mathrm{C} 3 \mathrm{bBbC} 3 \mathrm{~b}$ for instance have a half-life time of approximately $1.5-3 \mathrm{~min}$ at $37^{\circ} \mathrm{C}$ [17]. Also C5a itself is very shortlived and is cleaved rapidly into the more stable, though biologically still active acylation stimulating protein. However, the results of the study showed that the C5a content was not significantly affected by substrate degradation within the 30-90 min lasting test period.

After the test period of $90 \mathrm{~min}$ the clot formation process took significantly longer than after $30 \mathrm{~min}$. The prothrombin time was significantly increased $(p<0.05)$ after the $90 \mathrm{~min}$ lasting time period of testing in comparison to the $30 \mathrm{~min}$ lasting test cycle.

The fibrinogen content was measured based on the prothrombin time derived fibrinogen. In coagulation tests, the change in optical density during clot formation shows a progressive increase which is proportional to the fibrinogen concentration. The coagulometer exploited this change in optical density during the prothrombin time reaction to determine a prothrombin time derived (PT derived) fibrinogen concentration, by comparing the response of test plasma with that of a standard of known fibrinogen. The decreasing prothrombin time with increasing blood exposure time resulted in a decreasing fibrin 
formation from fibrinogen. For this reason the fibrinogen content in the plasma was significantly lower after 90 min of testing than after 30 min.

The thrombin time test allows evaluation of the step in the coagulation cascade, which is the conversion of fibrinogen into fibrin by thrombin, and we had expected also a positive correlation between the prothrombin time and the thrombin time. The platelet poor plasma which was tested before it was applied into the closed loop system the thrombin time was significantly faster after $30 \mathrm{~min}$ $(19.4 \pm 3.1 \mathrm{sec})$ than after $90 \mathrm{~min}(23.4 \pm 6.4 \mathrm{sec} ; p<0.05)$. But in contrast to the coagulation parameters prothrombin time and fibrinogen the thrombin time showed no significant difference after 30 and 90 min of testing in the closed loop system. This phenomenon is supposed to be caused by the closed loop system itself and might be mainly driven by the flow characteristic of the closed loop system, and the interactions of the closed loop system materials with thrombin inhibitors. Further tests will be performed to become more insight into these phenomena.

\section{Conclusion}

The study showed that in hemocompatibility testing a test period of $30 \mathrm{~min}$ is advantageous to a prolonged test period of $90 \mathrm{~min}$, if the tests are focused on the coagulation parameters prothrombin time and fibrinogen. For the coagulation parameter thrombin time and the measurement of the complement activation by the $\mathrm{C} 5 \mathrm{a}$ content comparable results can be expected within a time period of testing between $30 \mathrm{~min}$ and $90 \mathrm{~min}$.

\section{References}

[1] Wiliams DF (ed). Definitions in biomaterials, Elsevier, 1987.

[2] van Oeveren $\mathrm{W}^{1}$. Obstacles in haemocompatibility testing. Scientifica (Cairo) 2013;1-14.

[3] Braune, et al. Biointerphases 2013;8:33.

[4] Ratner BD. The catastrophe revisited: Blood compatibility in the 21st Century, Biomaterials 2007;28/34:5144-7.

[5] Seyfert UT, Jung F. Criteria and principles of in vitro hemocompatibility testing according to the ISO 10993 (4). Infus Ther Transfus Med 2000;27:317-22.

[6] Baier RE, Dutton RL. Initial events in the interaction of blood with a foreign surface. J Biomed Mater Res 1969;3:191-7.

[7] Seyfert U. In vitro hemocompatibility testing of biomaterials according to the ISO 10993 - 4. Biomol Eng 2002;19:91-96.

[8] Adcock D, et al., The effect of time and temperature variables on routine coagulation tests. Blood Coagul Fibrinolysis 1998;9:463-70.

[9] Rao LV, et al., Stability of prothrombin time and activated partial thromboplastin time tests under different storage conditions. Clin Chim Acta 2000;300:13-21.

[10] Breddin HK. Can platelet aggregometry be standardized? Platelets 2005;16:151-8.

[11] Cohen MG, et al. Insights into the inhibition of platelet activation by omega-3 polyunsaturated fatty acids: Beyond aspirin and clopidogrel. Thromb Res 2011;128:335-40.

[12] Anonymous, Ethical guidelines for publication in clinical hemorheology and microcirculation, Clin Hemorheol Microcirc 2010;44:1-2.

[13] Alström T, et al., Recommendations concerning the collection of reference values in clinical chemistry and activity report, Scand J Clin Lab Invest 1975;35:1-43.

[14] Baskurt OK, et al., New guidelines for hemorheological laboratory techniques, Clin Hemorheol Microcirc 2009;42:7597.

[15] Bach R, et al., Influence of a new monomeric nonionic radiographic contrast medium (Iobitridol-350 versus NaCl) on cutaneous microcirculation: Single-center, prospective, randomized, double-blind phase IV study in parallel group design, Microvasc Res 2000;60:193-200.

[16] Lemm W. (ed.), The reference materials of the European communities, Results of hemocompatibility tests. (1992), Springer-Science, Berlin.

[17] Cooper NR, Müller-Eberhard HJ. The reaction mechanism of human c5 in immune hemolysis. J Exp Med 1970;132: R775-93. 